\title{
A Social and Emotional Model for Obtaining Believable Emergent Behaviors
}

\author{
Javier Asensio, Marta Jiménez, Susana Fernández, and Daniel Borrajo \\ Planning and Learning research Group \\ Dept. of Computer Science, University Carlos III de Madrid \\ Av. de la Universidad 30. 28911 Leganés (Madrid), Spain \\ http://www.plg.inf.uc3m.es
}

\begin{abstract}
This paper attempts to define an emotional model for virtual agents that behave autonomously in social worlds. We adopt shallow modeling based on the decomposition of the emotional state in two qualities: valence (pleasantness or hedonic value) and arousal (bodily activation) and, also, for the agent personality based on the five factors model (openness, conscientiousness, extroversion, agreeableness and neuroticism). The proposed model aims to endow agents with a satisfactory emotional state achieved through the social actions, i.e. the development of social abilities. Psychology characterizes these social abilities for: using the language as a tool (verbal and nonverbal communication), being learned, producing reciprocal reward among the individuals involved in the communication and for depending on the individual features. We have implemented our model in the framework of a computer game, AILIVE, to show its validity.
\end{abstract}

Keywords: autonomous agents, behavior models, emotions and personality.

\section{Introduction}

Artificial Intelligence, as the branch of computer science that is concerned with the automation of intelligence behavior, has traditionally been interested in human behavior and its cognitive capabilities to emulate them or build intelligent systems. But, very few works have addressed the explicit representation and reasoning about important characteristics of humans that make them prefer some decisions over others: their emotion states and behaviors. Humans and emotions are highly intertwined, as well as the social and emotional behaviors. Emotions have been studied by different disciplines, such as psychology, neurobiology or philosophy (see 3 for an overview) without having reached a consensus on the emotional phenomena. However, many AI researchers agree with the functional hypothesis of Fridja [5 that emotions have the adaptive value of serving a purpose, called the functional view of emotions. Thus, some researchers want to endow artificial agents with emotions and thence with sociality, giving that emotions influence and shape the development of sociality as much as sociality influences and shaped the development of emotions. 
The latter years have witnessed some work in the field like the ones by: Cañamero discussing the ideas relative to the construction of emotional artifacts that have to interact in a social world [2]; or Silverman and his collaborators that focus on challenges to improve the realism of socially intelligent agents and on the attempt to reflect the state of the art in human behavior modeling [9]. And also, there are several interesting applications employing animated agents to enhance their effectiveness as tutors, sales persons, or actors, among other roles, pointed out in 8 .

This poses the need of designing and implementing emotional models for social interactions between artifacts, interacting with both, humans and other artifacts. The ability to provide formal approaches to emotions is a key aspect of current and future applications, that range from assistive technology, intelligent user interfaces, educational tools, automatic generation of scripts for films or tv shows, or games. This paper attempts to define such a social and emotional model. We have implemented it in the framework of a computer game to show its validity. The computer game is AI-LIVE [4, that is oriented towards the intensive use of AI controlled Bots. The game borrows the idea from the popular THE SIMS, where the player creates individual characters (units) that have significant autonomy, with their own drives, goals, and strategies for satisfying those goals, but where the human player can come in and stir things up by managing both the individual characters and their environment. Since our goal is to develop autonomous AI-driven for this kind of games, currently AI-LIVE only has automated players. The emotional model we propose here is based on the assumption that any emotional state can be decomposed into two qualities: valence (pleasantness or hedonic value) and arousal (bodily activation), borrowing the idea from [1. We prefer implementing such a shallow model, rather than a deep one as in [7. We also adopt shallow modeling for the agent personality based on the five factors model (openness, conscientiousness, extroversion, agreeableness and neuroticism) [6.

The proposed model aims to endow agents with a satisfactory emotional state achieved through social actions, i.e. the development of social abilities. In a first approach, the basic components of a model that complies with this idea will be: i) the agent psyche model that comprises agent's personality (the five factors), emotional state (valence and arousal) and social state (age, gender and place in the social hierarchy), ii) the social model for defining how every agent relates to the rest of agents and iii) the emotional engine that allows agents achieve satisfactory emotional states through communicative actions. In our design every personal relation is unidirectional and it involves only two actors (source and destination). This social link between agents is featured by an emotional state, being this state different from the actor's emotional state, but always under its influence; a kind of social relationship, can be based on age, blood or social status; the strength of the relationship, the stronger is the relation between two agents the more it will be affected by changes in the actors emotions and the start time for locating the beginning of the relationship. All these components are represented with numeric variables. The emotional engine 
defines how these relationships and emotions evolve over time. It consists of a set of formulae that compute the variation on the numeric variables involved in the model. In this paper, emotions as well as relations can only evolve by performing communicative actions, as talking or shouting. So far, we have only implemented the action tellLikeness where actors talk about their likeness of an specific object.

The remainder of the paper shows the empirical results that validate the model and the conclusions derived from the work, together with future research lines.

\section{Experiments and Results}

The experiments aim to assess the emotional model through the communicative action tellLikeness where an actor tells another his/her likeness of a particular object, $L_{S O}$, defined by a valence and arousal values. All the experiments were performed running the AI-LIVE system [4] with two rule-based AI clients: the sender (As), is the actor who talks and the receiver $(\mathrm{Ar})$, the actor who listens. We let As fulfils a certain amount of tellLikeness actions (20), always talking about the likeness of the same object, and we let Ar only listen to him/her (we ignore Ar actions when s/he gets the turn). When As tells his likeness to Ar we observe the following: i) As changes slightly the values valence and arousal of $L_{S O}$ transmitted to Ar, depending on his/her emotional state and his/her personality. ii) Ar also changes the values $L_{S O}^{t}$ received from As, depending on his/her personality. iii) The relationship between $\mathrm{Ar}$ and $\mathrm{As}, R$, changes its parameters strength, valence and arousal depending on the likeness difference, $L_{S O}^{t}-L_{R O}$, and on the social distance between both agents. iv) Ar changes his/her emotional state depending on the relationship changes above. And finally, Ar can change his/her likeness $L_{R O}$ depending on the new strength.

The independent variables in all the experiments were: 1. Agents (As and Ar) personality: (agreeableness, conscientiousness, extroversion, openness, neuroticism) all with values between 0 and 1 . We used three kinds of actors according with their personality: positive extreme $(1,1,1,1,0)$; negative extreme $(0,0,0,0,1)$; and, neutral $(0.5,0.5,0.5,0.5,0.5) .2$. Agents emotional state: (valence, arousal) (values between -1 and +1 ). We used three emotional states: positive extreme $(1,1)$, negative extreme $(-1,-1)$, and neutral $(0,0)$. 3 . The relationship between Ar and As, $R$ : kind, can be based on age, blood or social status, though we used age in the experiments; strength (values between 0 , if they are stranger, and 1); valence and arousal to represent the emotions that Ar arouse to As and startTime, we used 0.4. Agents social role: age (values between 0 and 100), though we used 20 in the experiments; sex (0 male or 1 female), we only used male in the experiments; and social status (values between 0 and 100), we used 5. 5. As likeness of the object $0, L_{S O}$. 6. Ar likeness of the object 0.

And we measured the transmitted likeness, as well as the variation on the strength of the relationship between the actors. Figure 1 shows the results of some of the experiments. We only plot the most significant ones. For example, the first graph reflects how As personality and his/her emotional state influence the 
communication. Normally, when an actor express his/her likeness of an object, the user never says the exact value of the valence and arousal, but changes them slightly. The closer his/her personality and his/her emotional state are to the positive extremes, the more he strengthens his/her likes. Something similar happens when Ar listens the likeness As is telling to him/her. Depending on his/her personality, s/he receives slight variations on the values of the valence and arousal transmitted by As. This variation only depends on the personality and emotional state of As but we have introduced a random value (between 0.5 and 1.3) that multiplies As emotional state and hence when As.x $=0$ the likeness transmitted is always the same: 1.01 when As personality is the positive extreme, 0.97 when is neutral and 0.94 when is the negative extreme. The As likeness to the object is fixed to 1 in all the cases.
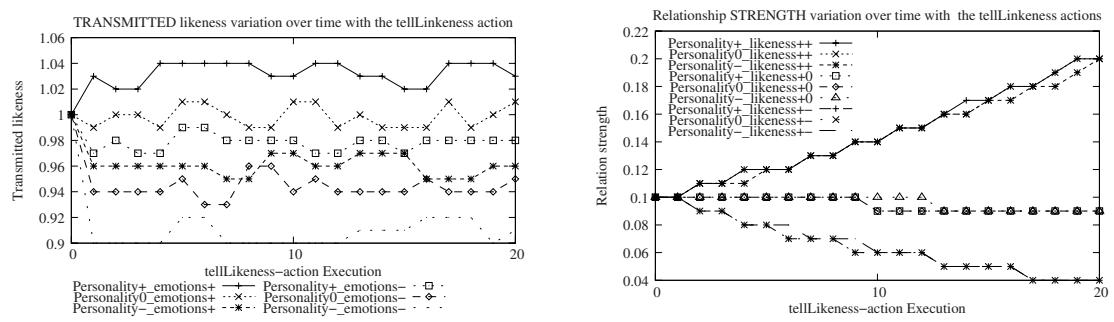

Fig. 1. Results for the tellLikeness action

The other graph reflects how the strength of the relationships changes depending on the initial affinity for the object. Initially, R.strength $=0.1$, R.valence $=$ 0, R.arousal $=0$ and the personality and emotional state of As are fixed to the positive extreme for all the experiments. We varied Ar personality from the positive extreme (Personality + ), neutral (Personality0) and negative extreme (Personality-) and the As and Ar likeness of the object. For example, likeness ++ means that the likeness emotions of both agents are the positive extreme, likeness +0 means that As likeness is the positive extreme and Ar likeness is neutral and likeness- means that both agents' likeness are the negative extreme. If the likeness for the object is similar for both agents, each communicative action strengthens the relationship. On the contrary, the strength decreases when their likeness are different, being the change proportional to the likeness unsimilarity, so the likeness+- graphs get lower strength values than the likeness +0 ones. The variations of Ar emotional state and likeness are proportional to this strength variation and we do not plot them for space reasons.

\section{Conclusions and Future Work}

This paper proposes an emotional and social model for obtaining believable emergent behaviors in autonomous agents. The initial hypothesis is that any 
agent can affect any other agent emotions by performing a communicative action as talking. A communicative action involves two agents and depends on the agents personality, their emotional states and the relationship between them. Therefore, the model must include a representation for all these objects, an implementation of the communicative actions and an emotional engine for evolving agents emotions. The emotional engine consists of a set of formulae that compute the variation on the numeric variables involved in the model. There are quite a few parameters that we have tuned experimentally. We have implemented all of this in the framework of a computer game inspired in the commercial game THE SIMS where each sim is an autonomous agent implemented with an AI technique. We have performed some experiments where an agent tells another its likeness about the same object and we have measured the variation of the emotions and relationship variables. The results show that the emotional engine evolves them in a coherent way.

In the future, we would like to augment the communication to include actions as gossip or shouting. Also, we would like to add actions where the agent not only communicates emotions but ideas and thoughts, as well. Furthermore, it would be interesting to generalize the emotional model to affect the whole decision making process of the agents.

\section{References}

1. Barrett, L.F.: Discrete emotions or dimensions? the role of valence focus and arousal focus. Cognition and Emotion 12, 579-599 (1998)

2. Cañamero, D.: Building emotional artifacts in social worlds: Challenges and perspectives. In: AAAI Fall Symposium on Emotional and intelligent II: The tangled knot of social cognition, pp. 22-30. AAAI Press, Menlo Park (2001)

3. Cornelius, R.: The Science of Emotion. Research and tradition in the psychology of emotion. prentice-hall, Upper Saddle River (1996)

4. Fernández, S., Adarve, R., Pérez, M., Rybarczyk, M., Borrajo, D.: Planning for an ai based virtual agents game. In: Proceedings of the Workshop AI Planning for Computer Games and Synthetic Characters in the ICAPS (2006)

5. Frijda, N.H.: Emotions in Robots. In: Comparative Approaches to Cognitive Science, pp. 501-516. MIT Press, Cambridge (1995)

6. McCrae, R., John, O.: An introduction to the five-factors model and its applications. Journal of Personality 60, 175-215 (1992)

7. Ortony, A., Clore, G.L., Collins, A.: The Cognitive Structure of Emotions. Syndicate of the University of Cambridge (1998) (in press)

8. Prendinger, H., Ishizuka, M.: Designing animated agents as social actors and dramatis personae for web-based interaction. Special Issue on Software Agent and its Applications (2002)

9. Silverman, B.G., Johns, M., Cornwell, J., O'Brien, K.: Human behavior models for agents in simulators and games: Part i: Enabling science with pmfserv. Departmental Papers (ESE) (2006) 